XXXI International Workshop on High Energy Physics:

Critical Points in the Modern Particle Physics

International Journal of Modern Physics: Conference Series

Vol. 47 (2018) 1860099 (6 pages)

(C) The Author(s)

DOI: $10.1142 /$ S2010194518600996

\title{
Generalized Parton Distributions and the Structure of the Hadrons
}

\author{
O. V. Selyugin \\ BLTPh, Joint Institut for Nuclear Research, Dubna, 141986, Russia \\ ab_selugin@theor.jinr.ru
}

Published 6 June 2018

\begin{abstract}
The dependence of the hadron interaction on its structure is examined in the framework of the generalized parton distributions (GPDs). The $x$ dependence of the GPDs is determined by the parton distribution functions (PDFs), which were obtained from the deep inelastic scattering. The analysis of the whole sets of experimental data on the electromagnetic form factors of the proton and neutron with taking into account many forms of PDFs, obtained by the different Collaborations, make it possible to obtain the special momentum transfer dependence of the GPDs. This permits us to obtain the electromagnetic and gravitomagnetic form factors of the nucleons. The impact parameter dependence of the proton and neutron charge and matter densities is examined. The elastic hadron scattering at high energies was analyzed in the framework of the model that takes into account both these form factors (electromagnetic and gravitomagnetic).
\end{abstract}

\section{Introduction}

The hadron diffraction processes at high energies give the valuable information about hadron structure. Especially it concerns the processes examined at the LHC with energy from $\sqrt{s}=7 \mathrm{TeV}$ up to $\sqrt{s}=14 \mathrm{TeV} .^{1}$ At these high energies the exchange of the mass Reggions with intercept less than unity can be neglected and the diffractive scattering is determined in most part by the cross-even massless Region - Pomeron and, possibly, the cross-odd Reggion -Odderon. The optic theorem links the imaginary part of the elastic scattering amplitude with the size of the total cross sections. The size and energy dependence of the total cross sections is connected intimately with the basic principle of quantum field theory and it is determined by the basic structure of the interaction hadrons, especially its radius of the interaction which is tightly connected with the hadron form factors. The first new data on the differential cross sections obtained at LHC do not coincide with any model predictions. This requires essential modernization of the phenomenological models. However, the new data on the total cross section, obtained by the two

This is an Open Access article published by World Scientific Publishing Company. It is distributed under the terms of the Creative Commons Attribution 4.0 (CC-BY) License. Further distribution of this work is permitted, provided the original work is properly cited. 
different Collaborations (TOTEM-CMS and group of ATLAS), again (as in the case of Tevatron) show a large difference which reaches up to $6 \mathrm{mb}$ at $\sqrt{s}=8 \mathrm{TeV}$. The sizes of the total cross sections are obtained from the analysis of the differential cross sections obtained at small momentum transfer with some model assumptions about the behavior of the hadron elastic scattering amplitude.

The hadron structure is represented in the structure of the scattering amplitude. It has to satisfy the basic principles of quantum field theory - casuality, analyticity, et cetera. The structure of the elastic scattering amplitude is represented by the imaginary and real parts and its energy and momentum transfer dependence. The basic properties require that the scattering amplitude should satisfy the integral dispersion relations. They tie the sizes and energy dependence of the real and imaginary parts of the elastic scattering amplitude. Hence, it is very important to determine the form and energy dependence of the real part and the ratio of the real to imaginary parts of the scattering amplitude - the value $\rho(s, t)$. To our regret the first experimental data obtained at the $\mathrm{LHC}$ at $\sqrt{s}=7 \mathrm{TeV}$ reveal some problems in the determination of the total cross sections and the size of $\rho(s=7) \mathrm{TeV}, t=0){ }^{2}$ The next experiment at $\sqrt{s}=8 \mathrm{TeV}$ shows that the nonlinear behavior of the differential cross sections at small momentum transfer exist at such large energy too. Such non-linear behavior, of course, is connected with hadron structure. In most part it can be determined by the contributions of the meson cloud surrounding the hadron. It leads to some problems in the determination of the total cross section and parameter $\rho(s, t=0) .^{2}$

The form factors can represent either the electromagnetic structure of the hadron or the matter distribution into the hadron. The different reactions can be related with the different form factors. It is very likely that the strong hadron-hadron interactions can be proportional to the matter distribution of the hadrons.

Both form factors can be obtained from the one function - Generalized parton distributions. The remarkable properties of the GPDs is that the integration of different momentum of GPDs over $x$ gives us different hadron form factors. ${ }^{3-5}$

The $x$ dependence of GPDs in most part is determined by the standard PDFs which are obtained by the different Collaborations from the analysis of the dip-inelastic processes. We can write the factorization form of GPDs $-\mathcal{F}_{i}=$ $P D F s_{i}(x) f(x, t) .{ }^{6}$ The main part of the $x$ dependence of GPDs is reflected in the standard parton distribution functions (PDFs) which are obtained from the analysis of the deep inelastic reactions by many different collaborations using the different forms of the $x$ dependence of the PDFs. However, to obtain the true form of the form factors, it is needed to know the true form of the function $f(x, t)$ which contains both the $x$ and the $t$ dependence.

\section{Hadron form factors}

On the basic of our analysis of many forms of the PDFs and practically all available experimental data on the electromagnetic form factors of the proton and neutron 
(the isotopic invariance can be used to relate the proton and neutron GPDs) the most preferable form of the PDFs was chosen. ${ }^{7}$

Taking $f(x)$ in the form $f_{u}(x)=\left[(1-x)^{2+\epsilon_{u}}\right] /\left[\left(x_{0}+x\right)^{m}\right]$ and $f_{d}(x)=(1+$ $\left.\epsilon_{0}\right)\left[(1-x)^{1+\epsilon_{d}}\right] /\left[\left(x_{0}+x\right)^{m}\right]$ we can obtain different parts of GPDs

$$
\begin{gathered}
\mathcal{H}^{u}(x, t)=q(x)^{u} e^{2 a_{H} f(x)_{u}{ }^{t}} ; \\
\mathcal{H}_{\Gamma}{ }^{d}(x, t)=q(x)^{d} e^{2 a_{H} f_{d}(x){ }^{t}} ; \\
\mathcal{E}^{u}(x, t)=q(x)^{u}(1-x)^{\gamma_{u}} e^{2 a_{E} f(x)_{u}{ }^{t}} ; \\
\mathcal{E}_{\Gamma}{ }^{d}(x, t)=q(x)^{d}(1-x)^{\gamma_{d}} e^{2 a_{E} f_{d}(x) t},
\end{gathered}
$$

The hadron form factors were obtained by the numerical integration over $x$ and then by fitting these integral results for the electromagnetic form factors by the standard dipole form with some additional parameters. It allows us to find separate contributions of the $u$ and $d$ quarks to the different parts of the electromagnetic form factors. ${ }^{7}$

The integration of the next moment of the GPDs gives us the mattter form factors, which was fitted by the also standard dypol form. It gives the size of the $\Lambda_{\text {grav. }}^{2}=1.6 \mathrm{GeV}^{2}$.

\section{Hadron form factors and elastic nucleon-nucleon scattering}

Note that the transition form factor $G_{M}^{*}(t)$ for the magnetic $N \rightarrow \Delta$ is depended only from the difference of $E_{u}(x, \xi=0, t)$ and $E_{d}(x, \xi=0, t)$. It is gives the unique possibility to check the momentum transfer dependence of spin depended part of GPDs. The relevant $G P D_{N \Delta}$ can be expressed in terms of the isovector GPD, taking into account $k_{v}=k_{p}-k_{n}=3.70$, yielding the sum rule ${ }^{8}$

$$
G_{M}^{*}(t)=\frac{G_{M}^{*}(t=0)}{k_{v}} \int_{-1}^{1} d x\left(E_{u}(x, \xi, t)-E_{d}(x, \xi, t)\right) .
$$

Figure 1a shows a sufficiently good coincidence with experimental data. It is confirmed that the form of the momentum transfer dependence of the $E(x, \xi, t)$ determined in our model is right.

It allows us to use both hadron form factors ( electromagnetic and gravitomagnetic) to build a high energy generalized structure model of the elastic nucleonnucleon scattering with minimum fitting parameters. ${ }^{9-11}$

The Born term of the elastic hadron amplitude can now be written as

$$
F_{h}^{\text {Born }}(s, t)=F_{I P}(\epsilon, s, t)\left(1+\frac{r_{1}}{\hat{s}^{0.5}}\right)+F_{3 g}^{\text {even }}(\epsilon, s, t)+F_{3 g}^{o d d}(\epsilon, s, t)\left(1+\frac{r_{2}}{\hat{s}^{0.5}}\right),
$$

with $\hat{s}=s e^{-i \pi / 2} / s_{0} ; s_{0}=4 m_{p}^{2} \mathrm{GeV}^{2}$. The intercept $1+\epsilon_{1}=1.11$ was chosen from the data of different reactions and was fixed by the same size for all terms of the Born scattering amplitude. The slope of the scattering amplitude has the standard logarithmic dependence on the energy $B(s)=\alpha^{\prime} \ln (\hat{s})$ with $\alpha^{\prime}=0.24 \mathrm{GeV}^{-2}$ and has some small additional term, ${ }^{10}$ which reflects the small non-linear behavior of 

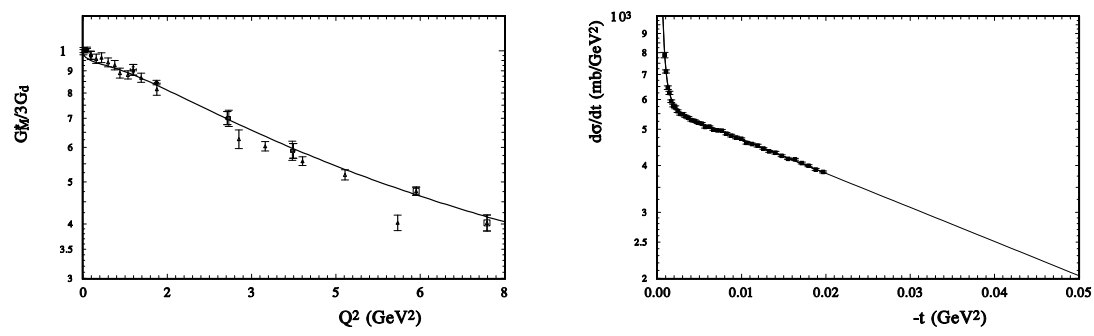

Fig. 1. a) [left] a) [left] Transition form factor $G_{M}^{*}(t)$ (line -the calculations in the framework of the model, points - the experimental data ${ }^{17}$ b) [right] Differential cross sections of the elastic $p p$ scattering are predicted by the HEGS model (line) and preliminary data of the TOTEM Collaboration. ${ }^{16}$

$\alpha^{\prime}$ at small momentum transfer. ${ }^{12}$ The final elastic hadron scattering amplitude is obtained after unitarization of the Born term. So, at first, we have to calculate the eikonal phase $\chi(s, b)=-\frac{1}{2 \pi} \int d^{2} q e^{i \vec{b} \cdot \vec{q}} F_{h}^{\text {Born }}\left(s, q^{2}\right)$, and then obtain the final hadron scattering amplitude

$$
F_{h}(s, t)=i s \int b J_{0}(b q) \Gamma(s, b) d b ; \quad \text { with } \quad \Gamma(s, b)=1-\exp [\chi(s, b)] .
$$

At large $t$ our model calculations are extended up to $-t=15 \mathrm{GeV}^{2}$. We added a small contribution of the energy independent part of the spin flip amplitude in the form similar to the proposed in ${ }^{13}$ and analyzed in. ${ }^{14}$ The model is very simple from the viewpoint of the number of fitting parameters and functions. There are no any artificial functions or any cuts which bound the separate parts of the amplitude by some region of momentum transfer. In the framework of the model the description of the experimental data was obtained simultaneously at the large momentum transfer and in the Coulomb-hadron region in the energy region from $\sqrt{s}=9 \mathrm{GeV}$ up to LHC energies. ${ }^{15}$ Figure $1 \mathrm{~b}$ presents the model predictions for $\sqrt{s}=13 \mathrm{TeV}$, that are in very good coincidence with the preliminary data presented at the conference BLOIS-17 (2017). ${ }^{16}$

\section{Structure of nucleons in the impact parameter representation}

The hadron structure is most obviously presented in the impact parameter representation. ${ }^{18,19}$ The charge density can be obtained by the integration of the GPDs over $x$ and $t$.

$$
\rho_{G_{E}}(b)=\sum_{q} e_{q} \int_{0}^{1} d x \int d^{2} q\left[H_{q}\left(x, \xi=0, q^{2}\right)+\tau E_{q}\left(x, \xi=0, q^{2}\right)\right] e^{i \vec{q} \vec{b}}
$$

where $\tau=\left(q / m_{p}\right)^{2}$. In the framework of our model of $t$-dependence of GPDs the calculation of both forms of the hadron charge distribution in the impact parameter 

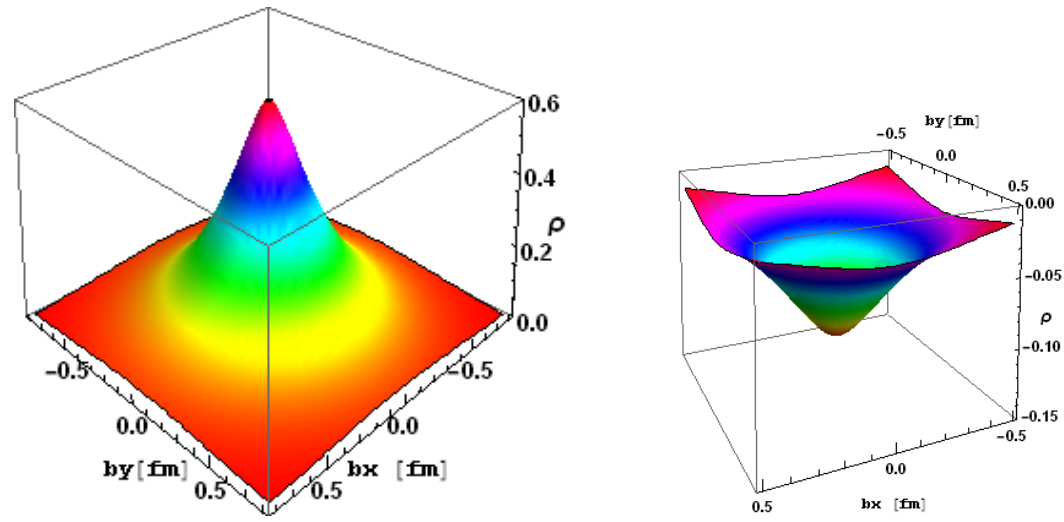

Fig. 2. a) [left] Charge density of $u$-quark b) [right] charge density of $d$-quark .
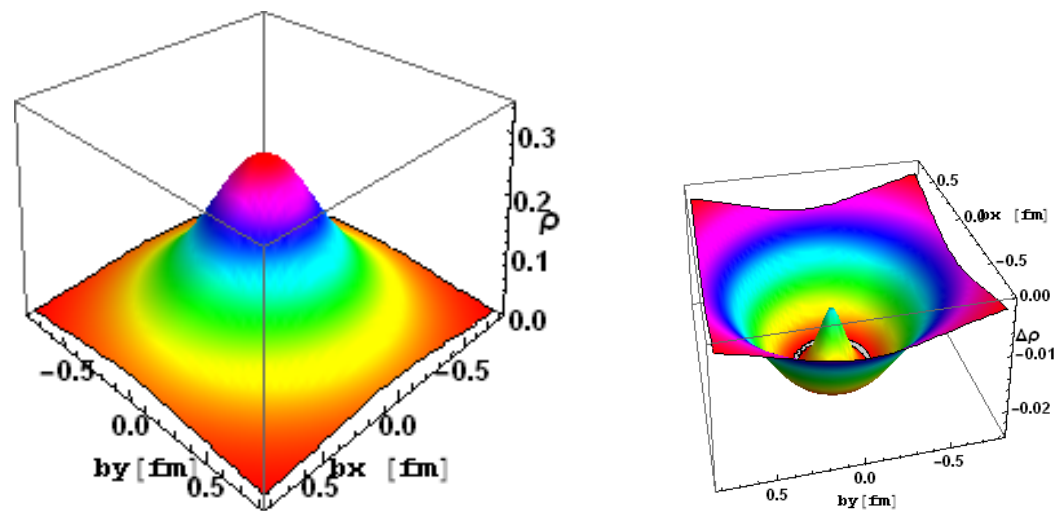

Fig. 3. a) [left] Difference between the charge density and matter dencity of $u$-quark; [right] difference between the charge density and matter density for $d$-quark.

representation can be carried out and, moreover, we can determine the separate contributions of $u$ and $d$ quarks (see Fig.2). It gives the possibility to compare the distribution of the electric charge and matter (that is, gravitational charge) in the nucleon which was obtained from the integration of the next momentum of GPDs. The difference of the charge density and the matter density of $u$ quark and $d$ quark for proton is shown in Fig.3(a,b).

\section{Conclusion}

The generalized parton distributions (GPDs) represent the basic properties of the hadron structure. The different properties of the hadron structure are reflected in the elastic scattering amplitude. One main part of it is the corresponding form factors of the nucleons. Our form of the momentum transfer dependence of the GPDs allow us to calculate the electromagnetic form factors, gravitomagnetic form factors, 


\section{O. V. Selyugin}

transition form factors, and Compton form factors. As a result, the description of various reactions is based on the same representation of the hadron structure. The results give good coincidence with the available experimental data. Especially it concerns the real Compton scattering and high energy elastic hadron scattering. The new high energy generalized structure (HEGS) model, based on the electromagnetic and gravitomagnetic form factors, gives a good quantitative description of the existing experimental data of the proton-proton and proton-antiproton elastic scattering in a wide region of the energy scattering and momentum transfer, including the Coulomb-hadron interference region, with the minimum number of fitting parameters. Its predictions describe quantitatively the experimental data obtained at the LHC at $\sqrt{s}=7$ and $\sqrt{s}=8 \mathrm{TeV}$, and are in good agreement with recent preliminary data at $\sqrt{s}=13 \mathrm{TeV}^{16}$ taking into account only statistical errors but with some small additional normalization coefficient. In the impact parameter representation the GPDs give us a separate contribution of the density of charge and matter distributions of the quarks.

\section{Acknowledgments}

The authors would like to thank V. Petrov for the invitation to take apart in the conference and J.-R. Cudell for fruitful discussion of some questions considered in the paper.

\section{References}

1. R. Fiore, L. Jenkovszky, R. Orava, E. Predazzi, A. Prokudin and O. Selyugin, Mod.Phys., A 24 (2009) 2551.

2. O.V. Selyugin, Nucl.Phys. A 922180 (2014).

3. D. Muller et al., Fortsch. Phys. 42, (1994) 101.

4. X.D. Ji, Phys. Lett. 78 , (1997) 610; Phys. Rev D 55 (1997) 7114.

5. A.V. Radyushkin, Phys. Rev. D 56, 5524 (1997).

6. O. Selyugin and O. Teryaev, Phys. Rev. D 79033003 (2009).

7. O.V. Selyugin, Phys. Rev. D 89093007 (2014).

8. M. Guidal et al., PhysRevD. D72 (2004) 054013.

9. O.V. Selyugin, Eur.Phys.J. C72, 2073 (2012).

10. O.V. Selyugin, Phys. Rev. D 91113003 (2015).

11. O. V. Selyugin, Nucl.Phys. A 90354 (2013).

12. O.V. Selyugin and J.-R. Cudell, in the Proceedings of the International workshop "Diffraction (2016), San Tesla di Acireale, Italy, September 02-08 (2016); arXiv:1611.04313.

13. M.V. Galynskii, E.A. Kuraev, Phys.Rev. D, 89 (2014) 054005.

14. O.V. Selyugin, PEPAN Letters, 13486 (2017).

15. O. V. Selyugin, Nucl.Phys. A 959116 (2017).

16. M. Deile (TOTEM Collaboration), talk in the Workshop on Diffraction in High-Energy Physics, Praha (Czech. Resp.), June 26-30 (2017).

17. F. Hagelstein, arxiv: 1710.00874.

18. M. Burkhardt, [hep-ph]/0509316.

19. H. Dahiya, A. Mukherjee, S. Ray, [hep-ph]/0705.3580. 